\title{
Cessação tabágica em indivíduos idosos - o que mudou e o que ainda falta fazer
}

Smoking cessation among older adults - what has changed and what still needs to be done

\section{Melanie Pires}

ACES Amadora, Administração Regional de Saúde de Lisboa e Vale do Tejo, Portugal melaniepires1392@gmail.com

Conflito de interesses: nada a declarar. Financiamento: nada a declarar.

Data de Submissão: 15/06/2021

Data de Aprovação: 10/07/2021 


\section{RESUMO:}

0 consumo do tabaco continua a ser uma das maiores ameaças de saúde pública a nível mundial. Os fumadores idosos apresentam maior carga tabágica do que os mais jovens, registando um maior número de complicações. Existem várias opções no apoio à cessação tabágica, no entanto, verifica-se uma lacuna no conhecimento quanto à intervenção mais efetiva em idosos. Foi realizada uma revisão rápida da literatura sobre cessação tabágica em idosos, principais abordagens terapêuticas, farmacológicas e não farmacológicas, e evolução ao longo dos últimos anos. A terapêutica com substitutos de nicotina, a existência de consultas intensivas, acessíveis e flexíveis, e um suporte social, são técnicas que parecem apresentar melhores resultados. Continua a ser evidente a existência de pouca literatura dirigida à cessação tabágica em adultos idosos, sendo por isso necessário o desenvolvimento de estudos científicos de elevada qualidade, de forma a reforçar os conhecimentos atuais e a prática clínica.

Palavras-chave: Cessação tabágica; idoso; tabagismo; tabaco

\section{ABSTRACT:}

Smoking remains one of the greatest public health threats worldwide. Elderly smokers have a greater consumption of tobacco and more related health problems than younger smokers. There are several options to support smoking cessation. However, there is still a lack of knowledge regarding the most effective interventions in elderly smokers. This rapid review of the literature focused on smoking cessation in the elderly, main therapeutic pharmacological and nonpharmacological approaches, and evolution over the last few years. Therapy with nicotine substitutes, the existence of intensive, accessible and flexible appointments, and social support, were the techniques that seemed to achieve better results. There is a lack of scientific literature on smoking cessation in elderly adults, which requires more high-quality scientific evidence, in order to improve current knowledge and clinical practice.

Keywords: Smoking cessation; elderly; smoking; tobacco 
https://doi.org/10.29073/jim.v2i2.429

\section{INTRODUÇÃO}

0 aumento da longevidade da humanidade, associado a uma redução da fertilidade carateriza uma das fases mais avançadas da transição demográfica. Neste momento, nos países de elevado rendimento (nos quais se integra Portugal), existe um aumento da proporção de população idosa face à população mais jovem (United Nations, 2019). Em Portugal, a população tem continuamente vindo a envelhecer, com mais idosos do que jovens a residir no país e atualmente os cidadãos com 65 ou mais anos representam 21\% da população (Ministério da Saúde, 2018). Com este aumento do número da população idosa foi necessário definir o termo "idoso", no entanto o mesmo não se encontra globalmente aceite. As Nações Unidas consideram que um idoso apresenta 65 ou mais anos (United Nations, 2019) enquanto a Organização Mundial de Saúde considera que um idoso será um indivíduo com 60 ou mais anos (World Health Organization, 2018). Em Portugal são considerados como idosos as pessoas com 65 ou mais anos (PORTDATA, 2021). Dadas as diferentes designações de pessoa idosa em termos de definição de idade, neste artigo optou-se por não categorizar por idade e apenas usar as diferentes terminologias gerais como idoso, pessoa idosa ou mesmo adulto idoso.

0 consumo do tabaco, em todas as suas formas de consumo ativo ou passivo, continua a ser uma das maiores ameaças de saúde pública a nível mundial, responsável pela morte de mais de 8 milhões de pessoas por ano (World Health Organization, 2020). Este é o segundo principal fator de risco, responsável por $13,6 \%$ dos casos de mortalidade precoce (Institute for Health Metrics and Evaluation, 2015). Em Portugal, o tabagismo contribui para uma morte a cada 50 minutos (Ministério da Saúde, 2018), estando relacionado com aproximadamente 11,7\% dos óbitos ocorridos no país, sobretudo em homens. As doenças associadas ao consumo de tabaco representam 121.643 anos de vida ajustados por incapacidade, dos quais 59\% são diretamente atribuíveis ao tabaco (Borges, et al., 2009).

Os fumadores idosos apresentam um consumo mais regular e em maior quantidade do que os fumadores mais jovens (Hall, et al., 2008). A prevalência de consumo de tabaco na Europa em 2010 era superior em homens (20,3\%, comparativamente a $13,1 \%$ em mulheres), sobretudo em países sem campanhas de intervenção dirigidas ao consumo de tabaco em idosos (Lugo, Vecchia, Boccia, Murisic, \& Gallus, 2013). Os idosos apresentam níveis mais elevados de dependência de nicotina, menor motivação para a cessação tabágica, menor apoio social, menor conhecimento e com mais dificuldade na eliminação metabólica da nicotina (Rimer, Orleans, Keintz, Cristinzio, \& Fleisher, 1990; Bryant, Bonevski, \& Paul, 2011; Hiscock, Judge, \& Bauld, 2011; Vangeli \& West, 2008; Molander, Hansson, \& Lunell, 2001). 
Apesar de ser aceite que o tabagismo traz consequências nefastas para a saúde em qualquer idade, algumas destas consequências afetam principalmente os mais idosos. Alguns estudos demonstraram que idosos fumadores apresentam valores de função cognitiva mais baixos do que idosos que nunca fumaram, juntamente com um acelerado declínio cognitivo (Elwood, et al., 1999; Mons, Schottker, Muller, Kliegel, \& Brenner, 2013; Stewart, Deary, Fowkes, \& Price, 2006; Ott, et al., 2004). Enquanto a doença pulmonar obstrutiva crónica (DPOC) apenas contribui ligeiramente para a mortalidade de fumadores jovens, no que respeita a fumadores com mais de 65 anos de idade e diagnosticados com DPOC a mortalidade tem um peso relevante (Burns, 2000). Cerca de $66,5 \%$ dos óbitos ocorridos por cancro do pulmão são atribuíveis ao consumo de tabaco, valor particularmente relevante acima dos 50 anos de idade. 0 consumo de tabaco representa também um importante fator de risco para o desenvolvimento de diabetes, doença isquémica e cerebrovascular, doença de Alzheimer, assim como neoplasia do esófago, laringe e bexiga (Institute for Health Metrics and Evaluation, 2015). Um artigo de revisão sistemática concluiu que o tabagismo é um preditor de agravamento da síndrome de fragilidade nos idosos, mostrando ser benéfica a cessação tabágica (Kojima, lliffe, \& Walters, 2015).

Apesar dos idosos poderem considerar que é demasiado tarde para deixar de fumar ou apresentar maior dependência nicotínica (Cox, 1993), os benefícios da cessação tabágica sobrepõem-se a essas eventuais limitações. No entanto, as motivações para a cessação tabágica num indivíduo idoso poderão ser diferentes das motivações para um adolescente ou jovem adulto (Tait, et al., 2007).

Quanto à eficácia dos diferentes tipos de intervenção em cessação tabágica aplicados a idosos, ainda existe pouca evidência científica, destacando-se a efetividade da utilização de substitutos de nicotina, intervenções não farmacológicas e intervenções mistas dirigidas à mudança comportamental (Cawkwell, Blaum, \& Sherman, 2015; Chen \& Wu, 2015).

Esta revisão tem por objetivo analisar a evolução temporal das evidências sobre técnicas de cessação tabágica aplicadas especificamente em indivíduos idosos, quer sejam farmacológicas ou não farmacológicas. Esta mantém-se como uma temática de importância crescente, tendo em conta a perspetiva de envelhecimento da população e as consequências para a saúde associadas ao consumo de tabaco, nas suas diversas formas de consumo. 
https://doi.org/10.29073/jim.v2i2.429

\section{METODOLOGIA}

Foi realizada uma revisão da literatura de artigos científicos indexados à base de dados PubMed, considerando a população idosa sujeita a intervenções de cessação tabágica (farmacológicas e não farmacológicas), tendo por comparação os resultados obtidos na população geral, nomeadamente quanto à efetividade da intervenção.

A estratégia de pesquisa teve por base a utilização dos medical subject headings (MeSH) em língua inglesa referentes à cessação tabágica e aos idosos conjugando os diferentes termos através dos carateres booleanos "AND" e "OR". Deste modo, utilizou-se a seguinte expressão de pesquisa, considerando diferentes terminologias válidas para os termos "cessação tabágica" e "idosos": (("Smoking Reduction"[Mesh]) OR ("Smoking Cessation Agents"[Mesh]) OR ("Tobacco Use Cessation"[Mesh]) OR ("Smoking Cessation"[Mesh]) OR ("Tobacco Use Cessation Devices"[Mesh])) AND (("Aged"[Mesh]) OR ("Aged, 80 and over"[Mesh])).

Apesar de a literatura cinzenta contribuir para o enriquecimento desta tipologia de artigos, neste trabalho apenas foram considerados artigos científicos indexados. 0 período de pesquisa decorreu num único período durante o mês de maio de 2021.

Para inclusão da literatura nesta revisão, estabeleceram-se os seguintes critérios: 1) artigos escritos em língua portuguesa ou inglesa; 2) publicações com o resumo e o artigo disponível; 3) artigos que contivessem os termos "cessação tabágica" e "idosos", nas suas variações já referidas, em língua inglesa, no título ou no resumo. A seleção dos artigos a rever foi efetuada em duas etapas: primeiro através da leitura dos títulos e resumos, com o objetivo de excluir os documentos que não cumprissem os critérios acima mencionados; posteriormente a seleção dos artigos com a leitura integral dos mesmos, tendo sido excluídos trabalhos que não abordassem a cessação tabágica como objeto de estudo principal no artigo (apenas considerando como intervenção preventiva) ou não considerassem uma análise específica do grupo etário considerado para a revisão.

0 processo de seleção, extração e revisão de dados na leitura integral dos artigos foi realizada apenas por uma revisora, tendo estes sido categorizados tendo em conta 0 ano de publicação do artigo, o limite de idade considerado para "idoso" ou "adulto idoso", as diferentes abordagens de intervenção em consultas de cessação tabágica no idoso (farmacológicas e não farmacológicas) e conclusões finais consideradas relevantes pelos autores. 


\section{RESULTADOS}

A pesquisa na base de dados PubMed, com recurso aos termos MeSH acima mencionados, resultou num total de 250 artigos iniciais. Destes, 75 foram excluídos após análise do título e 164 foram excluídos após leitura do resumo ou mediante a inexistência do mesmo. Deste modo, apenas 11 artigos foram selecionados para a leitura integral. Posteriormente, 3 artigos foram excluídos por não apresentarem como conteúdo principal a temática cessação tabágica no grupo dos idosos, ou por abordarem na sua maioria os efeitos do tabagismo neste grupo etário, não cumprindo deste modo os critérios para inclusão nesta revisão.

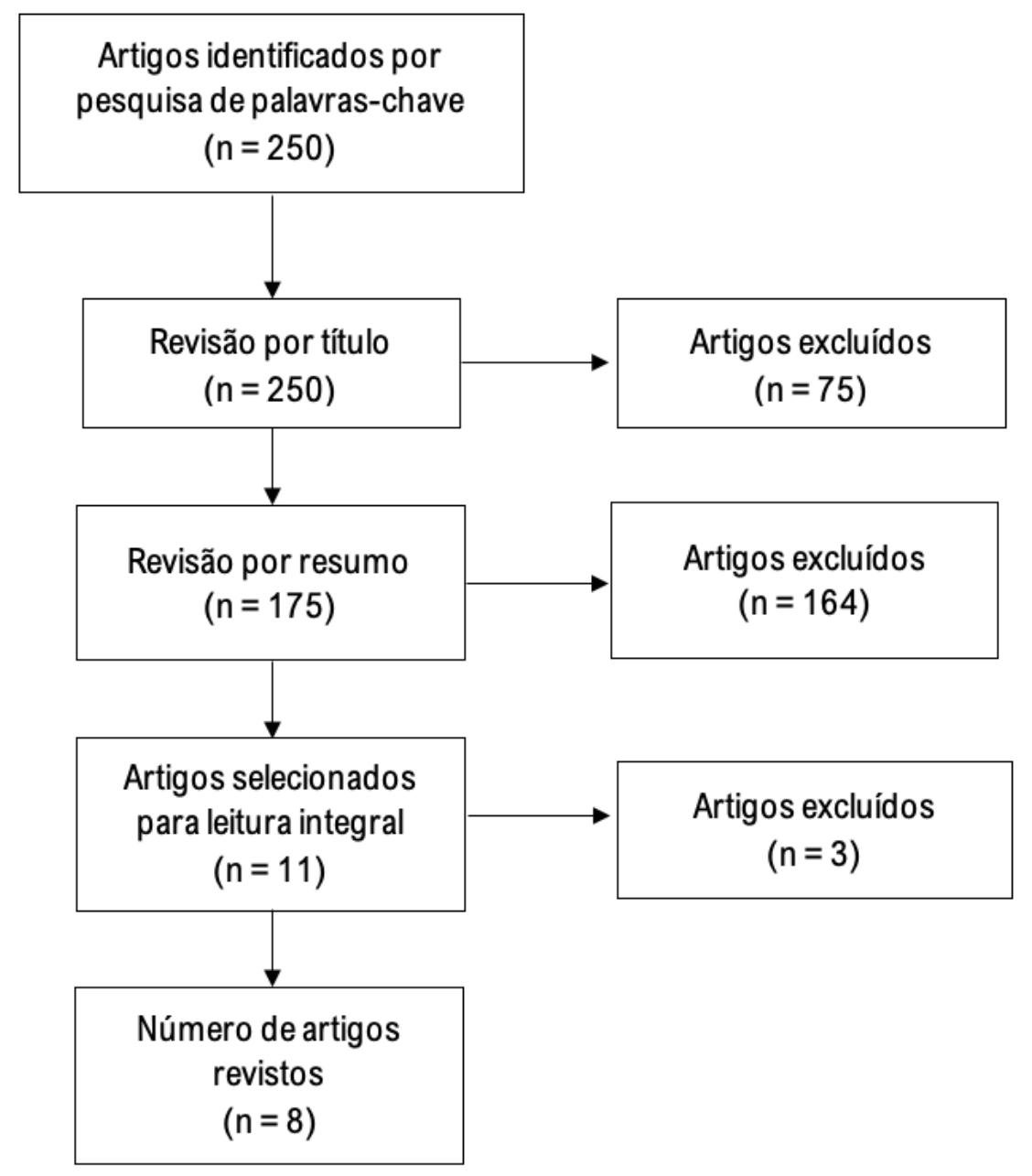

Figura1. Processo de seleção de artigos efetuada na revisão

No que respeita à definição de "adulto idoso" ou "idoso" em cada um dos artigos analisados, na sua maioria dos artigos revistos não está descrita a fundamentação inerente à seleção das idades consideradas. Estas variam desde 40 ou mais anos (Smith, et al., 2019) até 65 ou mais anos (Doolan \& Froelicher, 2008; Kerr, Watson, \& Tolson, 2002; Gourlay \& Benowitz, 1996). 


\section{https://doi.org/10.29073/jim.v2i2.429}

\section{Intervenções farmacológicas}

A terapêutica farmacológica utilizada na cessação tabágica foi sofrendo algumas alterações ao longo do tempo. Inicialmente surgiram os substitutos da nicotina, dos quais se destaca a bupropiona (antagonista dos recetores nicotínicos e inibidor da recaptação de noradrenalina e dopamina) e mais recentemente a vareniclina (agonista parcial dos recetores nicotínicos). No início dos anos 90 ainda não existiam estudos dirigidos à terapêutica de substituição nicotínica em idosos, mas suspeitava-se que não existiriam diferenças comparativamente ao uso das mesmas substâncias nos jovens, permitindo um elevado sucesso da cessação tabágica comparativamente ao placebo (Gourlay \& Benowitz, 1996). Verifica-se que literatura sobre farmacoterapia em idosos fumadores é escassa, com poucos estudos de qualidade e que apresentem amostra suficiente demonstrando a eficácia de novos fármacos como a bupropiona ou da vareniclina nos idosos fumadores, enquanto a eficácia do uso de substitutos de nicotina já se encontra bem demonstrada (Cawkwell, Blaum, \& Sherman, 2015).

\section{Intervenções não farmacológicas}

Existem várias metodologias não farmacológicas no apoio à cessação tabágica. No entanto, existe ainda uma falha no conhecimento quanto à melhor forma de intervenção neste grupo etário (Smith, et al., 2019). 0 apoio social pode ter uma importância mais relevante nos fumadores idosos em consultas de cessação tabágica comparativamente aos mais jovens (Zbikowski, Magnusson, Pockey, Tindle, \& Weaver, 2011; Appel \& Aldrich, 2003). 0 recurso a incentivos financeiros, a peer facilitators (sem termo de tradução direta para a língua portuguesa, sendo entendido como conselheiros que suscitam e medeiam a discussão sobre um determinado tema, oferecendo apoio emocional e orientação) e a um aconselhamento intensivo sobre cessação tabágica são outras metodologias descritas com resultados positivos, não estando ainda esclarecida se terá maior eficácia uma tipologia de intervenção individual ou de grupo. De salientar ainda que dependendo de quem realiza a intervenção ou consulta de cessação tabágica ao utente idoso (médico, enfermeiro ou outro profissional de saúde) poderá apresentar diferentes resultados, sendo esta área ainda alvo de investigação (Smith, et al., 2019). No entanto, num dos artigos selecionados é destacado o papel do enfermeiro para a obtenção de sucesso nas intervenções de cessação tabágica aos fumadores idosos (Kerr, Watson, \& Tolson, 2002).

\section{Oportunidades de intervenção em cessação tabágica nos idosos}

Os cuidados de saúde primários prestam cuidados de saúde a uma elevada proporção de utentes acima dos 65 anos, sendo por isso um local privilegiado para realizar intervenções breves em cessação tabágica aos utentes fumadores (Kerr, Watson, \& Tolson, 2002). No entanto, situações 
de hospitalização parecem ser um momento propício para intervenção de cessação tabágica nos utentes fumadores idosos, nomeadamente, na presença de problemas de saúde graves como um enfarte agudo do miocárdio (Gosney, 2001) ou na presença de alterações imagiológicas pulmonares relevantes (Zbikowski, Magnusson, Pockey, Tindle, \& Weaver, 2011).

Todos os artigos analisados referem que a consulta de cessação tabágica pode combinar uma vertente farmacológica com uma vertente não farmacológica, parecendo ser este o método que oferece melhores resultados. Um resumo das principais intervenções farmacológicas e não farmacológicas pode ser consultado no Quadro 1.

Quadro 1. Principais intervenções farmacológicas e não farmacológicas utilizadas na cessação tabágica.

\begin{tabular}{l|l}
$\begin{array}{l}\text { Intervenções farmacológicas em cessação } \\
\text { tabágica }\end{array}$ & $\begin{array}{l}\text { Intervenção não farmacológicas em cessação } \\
\text { tabágica }\end{array}$ \\
\hline $\begin{array}{l}\text { Substitutos de nicotina (adesivos, gomas, } \\
\text { pastilhas, entre outros) }\end{array}$ & $\begin{array}{l}\text { Aconselhamento individual ou em grupo } \\
\text { Peer facilitators }\end{array}$ \\
Bupropiona & $\begin{array}{l}\text { Incentivos financeiros } \\
\text { Vareniclina }\end{array}$ \\
& Consulta intensiva \\
Apoio social
\end{tabular}

\section{Desafios na cessação tabágica em idosos}

Apesar das diferentes intervenções atualmente disponíveis na cessação tabágica, os idosos apresentam características únicas, como o facto de fumarem há vários anos, estarem mais dependentes da nicotina e apresentarem menor adesão terapêutica, pelo que irão requerer estratégias diferentes das utilizadas nos indivíduos jovens (Cawkwell, Blaum, \& Sherman, 2015). No que respeita às dificuldades encontradas na população idosa, e que poderão comprometer 0 sucesso da cessação tabágica, estas passam por uma maior resistência a aconselhamentos sobre promoção de saúde (Gosney, 2001), valores de dependência de nicotina mais elevados, menor motivação e maior número de comorbilidades (Smith, et al., 2019; Gourlay \& Benowitz, 1996). No entanto, com as ferramentas necessárias e uma vez iniciado o processo, os fumadores idosos alcançam a abstinência em percentagens comparáveis aos mais jovens (Doolan \& Froelicher, 2008), apesar de manterem algum ceticismo, fatalismo e dúvida (Appel \& Aldrich, 2003). A evidência demonstra que parte da dificuldade em melhorar as intervenções de cessação tabágica 
https://doi.org/10.29073/jim.v2i2.429

nos idosos se deve à relutância dos profissionais de saúde, por eventual crença de eventuais consequências negativas resultantes deste processo, como seja o caso da redução da sensação de prazer (Kerr, Watson, \& Tolson, 2002).

\section{DISCUSSÃO}

0 tema da cessação tabágica na população idosa ainda se encontra pouco estudado e são necessárias revisões mais aprofundadas de literatura, mas também estudos originais de elevada qualidade, como ensaios clínicos randomizados.

Para este artigo de revisão a recolha dos dados da revisão integral do texto dos artigos selecionados baseou-se na seleção de informação sobre estratégias de cessação tabágica farmacológica e não farmacológica em indivíduos idosos com o intuito de analisar a evolução temporal da evidência científica nesta faixa etária. No entanto, apesar de ainda serem realizadas investigações científicas sobre este tema, existem poucas evidências recentes dirigidas a este grupo etário em específico. É igualmente importante ressalvar que a definição de idoso mostrou ser muito variável entre os diferentes artigos revistos, pelo que as conclusões retiradas para indivíduos de 40 ou mais anos poderão não ser totalmente válidas para indivíduos acima dos 65 anos.

\section{Exemplos de países com intervenções estruturadas por grupos específicos}

Alguns países já apresentam uma abordagem da cessação tabágica dirigida a grupos específicos, nos quais se integra o grupo das pessoas idosas fumadoras. É o caso da Escócia, que apresenta um trabalho de revisão sobre o tabaco em adultos idosos na população escocesa (ASH Scotland; NHS Health Scotland; The Scottish Government;, 2010). No referido trabalho, as conclusões a que os autores chegaram são em tudo semelhantes às conclusões a que se chegou neste artigo: a evidência existente demonstra os benefícios da cessação tabágica em idosos e a importância de estes serem priorizados em intervenções (Quadro 2.). A muitos idosos fumadores continua a não ser realizada uma intervenção para a cessação tabágica, como mostrado em projetos e iniciativas desenvolvidos na Escócia, o que os coloca numa desvantagem comparativamente à restante população. E as dificuldades encontradas para atingir o sucesso na abstinência tabágica passam por desafios como combater a dependência nicotínica e o uso do tabagismo como forma de ultrapassar a solidão, stress e como forma de ocupação. 
Quadro 2. Benefícios da cessação tabágica em idosos. Adaptado de ASH Scotland; NHS Health Scotland; The Scottish Government;2010.

\begin{tabular}{|c|c|}
\hline Redução da mortalidade & $\begin{array}{l}\text { - Em indivíduos que param de fumar } \\
\text { entre os } 65 \text { e os } 74 \text { anos } \\
\text { - Ganho de } 2 \text { anos na esperança média } \\
\text { de vida } \\
\text { - Redução de morte por causas } \\
\text { cardiovasculares } \\
\text { - Redução de morte por cancro (após } 15 \\
\text { a } 20 \text { anos de cessação tabágica) }\end{array}$ \\
\hline Mais qualidade de vida & $\begin{array}{l}\text { - } \text { Redução da incapacidade provocada } \\
\text { por doenças crónicas } \\
\text { - Proteção contra perda de } \\
\text { funcionalidade física } \\
\text { - Redução de complicações nos pós- } \\
\text { operatório, como cicatrização de } \\
\text { feridas }\end{array}$ \\
\hline Benefícios financeiros & $\begin{array}{l}\text { - Poupanças (principalmente para quem } \\
\text { tem baixos rendimentos) }\end{array}$ \\
\hline Benefícios para familiares e amigos & $\begin{array}{l}\text { - A exposição a tabagismo passivo } \\
\text { ("segunda-mão") aumenta o risco de } \\
\text { cancro do pulmão, doença cardíaca, } \\
\text { doenças respiratórias e crises de asma }\end{array}$ \\
\hline
\end{tabular}

Outro país que tem vindo a apresentar muitos trabalhos e com constante atualização na temática da cessação tabágica e a abordar as minorias e outros grupos específicos é a Austrália. 0 projeto "Tobacco in Australia - Facts and Issues" apresenta um capítulo sobre os fumadores idosos (Greenhalgh, Scollo, \& Winstanley, 2020), onde volta a ser reforçada a ideia de que a investigação existente atualmente ainda é limitada. À semelhança do referido nesta revisão, os profissionais de saúde acabam por realizar menos intervenções de cessação tabágica aos idosos por crenças não fundamentadas de que os idosos têm menos vontade e capacidade para terem sucesso na cessação tabágica, não respondendo com resultados positivos às intervenções, e que será errado retirar algo que traz momentos de prazer às suas vidas podendo até prejudicar a saúde do idoso fumador.

No que toca a Portugal, apenas foi encontrado um documento oficial da Direção-Geral da Saúde abordando sumariamente o consumo de tabaco na população, as suas consequências para a saúde, as principais medidas de prevenção, entre as quais a cessação tabágica, assim como um guia para a implementação de estratégias de cessação tabágica em consulta médica (Nunes, et al., 2007). Aqui também se podem ler as mesmas indicações já descritas anteriormente como os benefícios para a saúde serem semelhantes a outros grupos etários e a abordagem do tabagismo nos idosos ter as suas características próprias como assegurar o apoio social e psicológico e um 
https://doi.org/10.29073/jim.v2i2.429

acompanhamento proactivo. De realçar ainda que no "Programa Nacional - Prevenção e Controlo do Tabagismo 2019" são abordados em maior detalhe alguns grupos específicos como os jovens e as grávidas, mas não o grupo dos idosos (Nunes E. , 2019).

\section{Limitações}

A presente revisão apresenta algumas limitações, nomeadamente a realização do processo de revisão e redação ter sido realizado apenas por uma investigadora. Não foram incluídos neste trabalho estudos que não mencionavam nem no título nem no resumo a temática e a população tratadas neste artigo (cessação tabágica em idosos), apesar de poderem abordar no corpo do artigo, nem foi considerada literatura cinzenta na análise efetuada. Por outro lado, a pesquisa para revisão foi limitada a uma plataforma de pesquisa, apesar desta ser especificamente dedicada a artigos científicos na área médica. Apesar de inicialmente previsto, não foi possível estabelecer uma evolução temporal clara quanto à produção de novas evidências no que respeita às técnicas de cessação tabágica aplicadas ao grupo etário dos idosos, considerando a reduzida seleção de artigos após leitura integral. Foram identificados artigos científicos entre o início dos anos 90 e 0 ano de 2019, contudo, como vários artigos mencionam, estes não permitem retirar conclusões claras sobre a abordagem da cessação tabágica em indivíduos idosos.

Considerando a importância do tabaco como um dos principais fatores de risco para mortalidade precoce e o aumento da longevidade da população, sugere-se a realização de investigação adicional especificamente dirigida à população idosa, as suas motivações para consumir tabaco e para a cessação tabágica, assim como estudos comparativos entre diferentes grupos etários sujeitos a intervenções de cessação tabágica.

\section{CONCLUSÃO}

Apesar da cessação tabágica ser uma intervenção clínica discutida há várias décadas, existe pouca literatura científica especificamente dirigida a indivíduos idosos.

Dadas as vantagens em deixar de fumar, independentemente da idade da pessoa, deve-se considerar a maior dependência de nicotina, a menor motivação, maior número de comorbilidades da população idosa nas diversas abordagens adotadas. Estas características dos fumadores idosos parecem requerer estratégias de intervenção especificamente dirigidas a este grupo etário, diferentes das utilizadas nos mais jovens. Apesar de disponíveis intervenções farmacológicas e não farmacológicas no apoio à cessação tabágica (uso de substitutos de nicotina ou existência de 
consultas intensivas e de apoio social), nesta esta revisão conclui-se que é necessário o desenvolvimento de mais estudos científicos de elevada qualidade, especificamente dirigidos à cessação tabágica em idosos, de forma a reforçar os conhecimentos atuais e a prática clínica.

\section{Perspetivas futuras}

Alguns profissionais de saúde poderão realizar menos intervenções dirigidas à sessão tabágica devido a crenças de uma menor efetividade em idosos. Com o surgimento de evidência científica sobre o melhor método de intervenção em cessação tabágica nos idosos, será importante encorajar a formação de mais profissionais de saúde nesta área, particularmente daqueles que contactam mais frequentemente com indivíduos idosos, e por isso capazes de intervir eficazmente na cessação tabágica. 
https://doi.org/10.29073/jim.v2i2.429

\section{BIBLIOGRAFIA}

Appel, D. W., \& Aldrich, T. K. (2003). Smoking cessation in the elderly. Clinics in Geriatric Medicine, 77-100.

ASH Scotland; NHS Health Scotland; The Scottish Government;. (2010). Tobacco and Older Adults: A Literature Review.

Borges, M., Gouveia, M., Costa, J., Pinheiro, L., Paulo, S., \& Carneiro, A. (2009). Carga de doença atribuível ao tabagismo em Portugal. Revista Portuguesa de Pneumologia, 951-1004.

Bryant, J., Bonevski, B., \& Paul, C. (2011). A survey of smoking prevalence and interest in quitting among social and community service organisation clients in Australia: a unique opportunity for reaching the disadvantaged. BMC Public Health, 1-6.

Burns, D. (2000). Cigarette smoking among the elderly: disease consequences and the benefits of cessation. American Journal of Health Promotion, 357-361.

Cawkwell, P. B., Blaum, C., \& Sherman, S. E. (2015). Pharmacological Smoking Cessation Therapies in Older Adults: Review of the Evidence. Drugs Aging.

Chen, D., \& Wu, L. (2015). Smoking cessation interventions for adults aged 50 or older: A systematic review and meta-analysis. Drug and Alcohol Dependence, 14-24.

Cox, J. (1993). Smoking cessation in the elderly patient. Clinics in Chest Medicine, 423-428.

Doolan, D. M., \& Froelicher, E. S. (2008). Smoking Cessation Interventions and Older Adults. Progress in Cardiovascular Nursing, 119-127.

Elwood, P., Gallacher, J., Hopkinson, C., Pickering, J., Rabbitt, P., \& Stollery, B. (1999). Smoking, drinking, and other life style factors and cognitive function in men in the Caerphilly cohort. Journal of Epidemiology and Community Health, 9-14.

Gosney, M. (2001). Smoking Cessation. Gerontology, 236-240.

Gourlay, G. S., \& Benowitz, L. N. (1996). The Benefits of Stopping Smoking and the Role of Nicotine Replacemente Therapy in Older Patients. Drugs \& Aging, 8-23.

Greenhalgh, E., Scollo, M., \& Winstanley, M. (2020). Tobacco in Australia: Facts and issues. Cancer Council Victoria. Obtido de www.TobaccolnAustralia.org.au

Hall, S., Humfleet, G., Gorecki, J., Munoz, R., Reus, V., \& Prochaska, J. (2008). Older versus younger treatmente-seeking smokers: differences in smoking behaviour, drug and alcohol use, and psychosocial and physical functioning. Nicotine Tob Res, 463-470. 
Hiscock, R., Judge, K., \& Bauld, L. (2011). Social inequalities in quitting smoking: what factors mediate the relationship between socioeconomic position and smoking cessation? Journal Public Health, 39-47.

Institute for Health Metrics and Evaluation. (2015). GBD Compare. (University of Washington) Obtido em 14 de Junho de 2021, de http://vizhub.healthdata.org/gbd-compare

Kerr, S. M., Watson, H. E., \& Tolson, D. (2002). Older people who smoke: why nurses should help them to stop. British Journal of Nursing, 1012-1018.

Kojima, G., lliffe, S., \& Walters, K. (2015). Smoking as a predictor of frailty: a systematic review. BMC Geriatrics, 1-7.

Lugo, A., Vecchia, C., Boccia, S., Murisic, B., \& Gallus, S. (2013). Patterns of Smoking Prevalence among the Elderly in Europe. International Journal of Environmental Research and Public Health, 10(9), 4418-4431.

Ministério da Saúde. (2018). Retrato da Saúde. Portugal.

Molander, L., Hansson, A., \& Lunell, E. (2001). Pharmacokinetics of nicotine in healthy elderly people. Clinical Pharmacology and Therapeutics, 57-65.

Mons, U., Schottker, B., Muller, H., Kliegel, M., \& Brenner, H. (2013). History of lifetime smoking, smoking cessation and cognitive function in the elderly population. European Journal of Epidemiology, 823-831.

Nunes, E. (2019). Programa Nacional para a Prevenção e Controlo do Tabagismo. Lisboa: DireçãoGeral da Saúde.

Nunes, E., Candeias, A., Mendes, B., Pardal, C., Fonseca, J., Rebelo, L., . . Vinagre, S. (2007). Cessação Tabágica - Programa-tipo de actuação. Direcção-Geral da Saúde. Gradiva.

Ott, A., Andersen, K., Dewey, M., Letenneur, L., Brayne, C., \& Copeland, J. (2004). Effect of smoking on global cognitive function in nondemented elderly. Neurology, 920-924.

PORTDATA. (2021). Indicadores de envelhecimento. Obtido de https://www.pordata.pt/Site_Widgets/MetalnfoPrint.aspx?MicrolDs=526\&ExpandMeta=True\&Wor kMicroVersionld=0\&Languageld=1\&DataBaseModeld=1\&GeographicGroupld_Level1=361\&Geogr aphicGroupld_Level2=0

Rimer, B., Orleans, C., Keintz, M., Cristinzio, S., \& Fleisher, L. (1990). The older smoker. Status, challenges and opportunities for intervention. Chest, 547-553. 


\section{https://doi.org/10.29073/jim.v2i2.429}

Smith, P., Poole, R., Mann, M., Nelson, A., Moore, G., \& Brain, K. (2019). Systematic review of behavioural smoking cessation interventions for older smokers from deprived backgrounds. BMJ Open, 1-10.

Stewart, M., Deary, I., Fowkes, F., \& Price, J. (2006). Relationship between lifetime smoking, smoking status at older age and human cognitive function. Neuroepidemiology, 83-92.

Tait, R., Hulse, G., Waterreus, A., Flicker, L., Lautenschlager, N., \& Jamrozik, K. (2007). Effectiveness of a smoking cessation intervention in older adults. Addiction, 148-155.

United Nations. (2019). Global Issues. Obtido de Ageing: https://www.un.org/en/globalissues/ageing

United Nations. (2019). World Population Ageing.

Vangeli, E., \& West, R. (2008). Sociodemographic differences in triggers to quit smoking: findings from a national survey. Tobacco Control, 410-415.

World Health Organization. (5 de Fevereiro de 2018). Ageing and health. Obtido de https://www.who.int/news-room/fact-sheets/detail/ageing-and-health

World Health Organization. (27 de Maio de 2020). Tobacco. Obtido de https://www.who.int/newsroom/fact-sheets/detail/tobacco

Zbikowski, M. S., Magnusson, B., Pockey, R. J., Tindle, A. H., \& Weaver, E. K. (2011). A review of smoking cessation interventions for smokers aged 50 and older. Maturitas, 131-141. 